\title{
Vom Konflikt zur Kooperation? \\ Aktuelle Fragen internationaler Kommunikationspolitik
}

von Benno Signitzer

Eine der interessantesten Entwicklungen in den internationalen Beziehungen der letzten Jahre ist der dramatische Bedeutungszuwachs von Fragen der internationalen Kommunikation. Eine kaum mehr zu überschauende Anzahl von internationalen Konferenzen, Symposien, Workshops und Publikationen sind den „Strukturen und Prozessen, die den Medien- und Kommunikationsaktivitäten zwischen Ländern und Kulturen zugrunde liegen"l, gewidmet. Zahlreiche zwischenstaatliche internationale Organisationen (wie z.B. UNO und UNESCO) ${ }^{2}$ haben nicht unwesentliche Teile ihrer Tätigkeitsfelder diesen Fragen zugewiesen - ganz zu schweigen von den vielen medien- bzw. kommunikationsspezifischen nichtstaatlichen Organisationen (den sog. NGO's) und den professionellen Vereinigungen, die die internationale Dimension ihrer Aufgabenbereiche zunehmend in den Vordergrund stellen. Es ist daher kaum eine Übertreibung, wenn der Direktor des Londoner International Institute of Communications - auch im Hinblick auf Fragen der internationalen Kommunikation - jüngst feststellte: "During the last few years, issues of communication have been moved to the centre of the political stage. For the first time we have seen governments fall over broadcasting policy, and a major international organisation, UNESCO, almost being torn apart over mass media regulation. And we continue to commit intellectual and financial resources to what appears to be an endless series of conferences with an equally limitless number of resolutions, recommendations, controversies und opinions". 3

Es würde den Rahmen dieser Ausführungen sprengen, im einzelnen die Gründe für diese Entwicklung zu analysieren. Sie könnten aber in Zusammenhang gebracht werden mit tiefgreifenden gesellschaftlichen Strukturveränderungen (vor allem in Industrieländern), die mit Schlagworten wie „Postindustrielle Gesellschaft” oder "Informationsgesellschaft" umrissen werden ${ }^{4}$. Ploman identifiziert diesbezüglich drei Faktoren, die ihm von besonderer Wichtigkeit erscheinen:

- the development of new technology and the impact of the communications revolution;

- the transformation of international relations and the international system;

- the attacks against the traditional concepts of freedom of information and free flow of information and the criticism that they serve as justifications for practices reflecting dominance and inequality; and the emergence of such new concepts as a new world information and communications order which cut across old categories and introduce new perspectives 5 .

Die Bedeutung der diesbezüglichen internationalen Debatten und der damit verbundenen Aktivitäten sind in den letzten Jahren auch von kirchlichen Organisationen erkannt worden. Im deutschsprachigen Raum beschäftigt sich die vorliegende Zeitschrift „Communicatio Socialis” in der letzten Zeit vermehrt mit kirchlichen,

Dr. Benno Signitzer, Ph.D., ist Univ.-Assistent am Institut für Publizistik und Kommunikationswissenschaft der Universität Salzburg. 
aber auch allgemeinen Aspekten von Entwicklungsländerpublizistik und internationaler Kommunikation ${ }^{6}$, und auch die Publikationen des Gemeinschaftswerkes der evangelischen Publizistik haben neuerdings Fragestellungen dieser Art aufgegriffen $^{7}$. Die deutschsprachige Publizistik- und Kommunikationswissenschaft hat dieses Forschungsgebiet für lange Zeit vernachlässigt; erst in allerletzter Zeit sind einige Beiträge dazu erschienen ${ }^{8}$.

Die Absicht der folgenden Ausführungen kann es nicht sein, die Gesamtheit der Problemfelder der internationalen Kommunikation analytisch zu erfassen und zu bewerten. Dies für den deutschsprachigen Raum zu leisten, müßte einer weitaus größeren Arbeit vorbehalten bleiben. Im folgenden werden daher Thematik und Anspruch eingeschränkt auf

a) einige ausgewählte Fragen der Kommunikationspolitik (insbesondere Medienentwicklung, Empfehlungen der MacBride-Kommission, Rolle der Kommunikationswissenschaft) in ihrer Evolution in internationalen Gremien; und

b) Entwicklungen in allerjüngster Zeit, insbesondere im ersten Halbjahr 1980.

Die Konzentration auf aktuelle Ereignisse hat drei Gründe: erstens hat es im Jahre 1980 eine Reihe von Entwicklungen von größerer Tragweite gegeben; zweitens hat m.E. die politische Debatte um Fragen der internationalen Kommunikation eine neue Dimension erlangt (nämlich durch den Schritt von Diskussion und Polemik zu Aktion und Kooperation); und drittens werden einige der im ersten Halbjahr 1980 vorbereiteten Schritte bei der kommenden 21. UNESCO-Generalkonferenz in Belgrad im Herbst 1980 zur Debatte stehen und möglicherweise zu weitreichenden Entscheidungen führen. Aus dem Gesagten ergibt sich, daß die folgenden Ausführungen in weiten Teilen Berichtscharakter haben werden und Einschätzungen bzw. Bewertungen als vorläufig zu betrachten sind, da sie von der weiteren Entwicklung schon in kurzer Zeit durchaus überholt sein könnten.

Fragen der internationalen Kommunikation werden auf politischer Ebene noch weitgehend ohne die Unterstützung einer auf breiter Basis geführten öffentlichen Diskussion behandelt (zumindest auf Österreich trifft dies zu); die folgenden Ausführungen sind als informierender Beitrag zu einer solchen Diskussion zu verstehen - auch im Hinblick auf die Belgrader UNESCO-Generalkonferenz im Herbst 1980. Die umfangreichen Anmerkungen enthalten eine Reihe von weiteren Hinweisen.

\section{Der Rabmen der Debatte}

Es gehört zu den Charakteristika der Debatten um Fragen der internationalen Kommunikation, daß sie sich weniger auf bilateraler, sondern vielmehr auf multilateraler Ebene, insbesondere in großen internationalen Organisationen, entwickeln. Dies hat eine Reihe von Implikationen:

1. politische, weil sich in den meisten weltweiten Organisationen (z.B. UNO, UNESCO) die sog. „Neue Mehrheit” der Entwicklungsländer immer fester etabliert und oft über ideologische Trennlinien hinweg eine - durchaus nicht nur rhetorische - Interessenidentität angestrebt und oftmals auch erfolgreich formuliert wird. In Fragen der internationalen Kommunikation drückt sich diese neue Situation in der Forderung nach einer neuen Weltinformations- und -kommunika- 
tionsordnung aus ${ }^{9}$, die ausgewogenere Kommunikationsbeziehungen zwischen Industrie- und Entwicklungsländern anstrebt - sowohl im Hinblick auf Kommunikationsflüsse als auch Kommunikationsinfrastrukturen;

2. inhaltliche, weil die "Neue Mehrheit" Arbeitsprogramm und Ressourcenverteilung der internationalen Organisationen im Sinne der Bedürfnisse der Entwicklungsländer zu gestalten imstande ist. Die Interessen der Entwicklungsländer sind somit grundätzlich in einem multilateralen Kontext besser gewahrt als in bilateralen Beziehungen, die oft einseitige Abhängigkeit vom stärkeren Partner implizieren;

3. organisatorisch-strukturelle, weil die Strukturen der meisten internationalen Organisationen bzw. der Beziehungen zwischen diesen sich im Laufe der Zeit zwar neuen Mehrheitsverhältnissen anpassen konnten, nicht jedoch immer neuen Aufgabenstellungen wie etwa Kommunikation als integrativer Problembereich. In ihrer Unfähigkeit, neue Organisationsformen für neue Probleme und Herausforderungen zu entwickeln, spiegeln internationale Bürokratien jedoch oft nur innerstaatliche Kompetenzprobleme für Fragen der Kommunikationspolitik wider.

In dem Maße, in dem internationale Kommunikationspolitik weitgehend internationale Gremienpolitik geworden ist, ist sie naturgemäß von dem beeinflußt, was Ploman im obigen Zitat "the transformation of international relations and the international system" nennt, was wiederum aufs engste mit dem Entkolonialisierungsprozeß einerseits und der daraus entstandenen Forderung nach einer "Neuen Weltordnung" (z.B. Neue Weltwirtschaftsordnung) andererseits zusammenhängt. Daraus ergibt sich, daß Fragen der internationalen Kommunikation nicht isoliert betrachtet werden können von allgemeinen Problemen der internationalen Politik und Wirtschaft. Westliche bzw. westlich orientierte Staaten sind gerade im Begriffe, diesen grundsätzlichen Zusammenhang zu erkennen, und frühere Positionen einer isolierten Betrachtungsweise, die sie selbst in die Isolation geführt haben, zu revidieren. Dieser Umstand hat m.E. u.a. auch dazu beigetragen, daß die Stoßrichtung der internationalen Debatte seit kurzer Zeit in vermehrtem Maße in Richtung Kooperation geht. Während die Atmosphäre der Debatte bislang von einer Konfrontation zwischen Industrie- und Entwicklungsländern bestimmt war (und sich häufig an Begriffen wie ,free flow of information” oder „Zensur” entzündete), ist derzeit (Mitte 1980) ein Trend zu praktischen Maßnahmen und Kooperation zu beobachten. Rolle und spezifische Interessenlage kleinerer Industriestaaten in diesem Konflikt (und ein Konflikt ist es noch immer; die UNESCO-Generalkonferenz in Belgrad kann durchaus die antagonistische Komponente gegenüber der kooperativen wieder stärker akzentuieren) wurden bislang noch nicht ausreichend analysiert. Während die offiziellen „Kommmunikationsaußenpolitiken” dieser Staaten sich bisher - basierend auf den herrschenden Werten der jeweiligen innerstaatlichen Kommunikationsordnungen - ziemlich nahtlos am Verhalten ihrer großen „Industriestaaten-Brüder" orientierten, scheint sich ansatzweise eine Überprüfung eigener Interessenlagen abzuzeichnen. So haben etwa Kanada und Schweden bei den Beratungen über die Regulierung des Satellitendirektfernsehens im Weltraumausschuß der UNO von Anfang an gemeinsam eine vermittelnde Kompromißposition zwischen den westlichen, östlichen und Dritte-Welt-Auffassungen entwickelt ${ }^{10}$. 
Einer der wesentlichen Fortschritte, die die internationale Debatte über Kommunikationsfragen in den letzten Monaten erzielt hat, ist die Hinwendung zu konkreten Problemen der Medien- und Kommunikationsentwicklung. Damit soll aber nicht gesagt sein, daß die frühere „Diskussions- und Polemikphase” (etwa seit 1974) ganz ohne Wert war.

Vom Interessenstandpunkt der Entwicklungsländer waren die heftigen Angriffe auf die bestehende Macht- und Ressourcenverteilung und die damit verbundene Polemik gegen das Prinzip der internationalen Informationsfreiheit, das als Legitimationsvehikel zur Aufrechterhaltung ungleichgewichtiger Verhältnisse im Weltkommunikationssystem kritisiert wurde, notwendig, um überhaupt erst die Aufmerksamkeit auf eben diese Situation zu lenken. Schwere Auseinandersetzungen bei der UNESCO-Generalkonferenz 1976 in Nairobi über die Mediendeklaration der UNESCO haben schließlich zu einem Kompromiß in Form eines "package deal"geführt, in dem die westlichen Industriestaaten den Entwicklungsländern umfangreiche Finanzhilfe zur Entwicklung ihrer Medien in Aussicht stellten, die ihrerseits Forderungen nach weitgehenden Einschränkungen des Prinzips der internationalen Informationsfreiheit zurückstellten, so daß 1978 die Mediendeklaration einstimmig verabschiedet werden konnte ${ }^{11}$.

Als weiterer Teil dieses "package deal" ist die Einsetzung der International Commission for the Study of Communication Problems (MacB ride-Kommission) zu sehen, die 1977 ihre Arbeit aufnahm, 1978 einen Zwischenbericht und Anfang 1980 den Endbericht vorlegte, der im August desselben Jahres in Buchform unter dem Titel "Many Voices, One World"12 erschienen ist (siehe unten).

Die Entwicklungsländer und die UNESCO haben das Angebot der westlichen Industriestaaten aufgegriffen, und es ist in der Folge zu Entwicklungen mit möglicherweise weitreichenden Konsequenzen gekommen. Der UNESCO-Generaldirektor hat im April 1980 eine große Regierungskonferenz über "Co-operation on Activities, Needs and Programmes for Communication Development" nach Paris einberufen, an der 122 Staaten, 12 internationale Organisationen und 21 NGO's teilnahmen. Diese Konferenz, die erste ihrer Art, hat die ganze Bandbreite des Problemfeldes Kommunikation und Entwicklung erhellt: einerseits wurden konzeptionelle Widersprüche und politische Interessensgegensätze deutlich, andererseits aber auch konstruktive Möglichkeiten für echte Kooperation aufgezeigt. Die gesammelten Arbeitspapiere, Resolutionsentwürfe und Delegiertenaussagen sind eine Fundgrube für Kommunikationswissenschaftler, die sich im Detail komparativ mit dem kommunikationspolitischen Ist-Zustand in diesem Themenbereich Anfang der 80 er Jahre befassen wollen. Für $Z$ wecke dieses Überblickes genügt es, auf einige wenige Aspekte einzugehen.

Eine - banale - Erkenntnis ist die: Es ist leichter, alte, nachweisbar überholte, Konzepte aufzugeben, als neue zu entwickeln. Dies wurde auf der Pariser Konferenz in zweifacher Hinsicht deutlich: in der Frage der Weite des Kommunikationsbegriffes und der Einschätzung der Kommunikationstechnik und der damit verbundenen Entwicklungsmodelle. Was den Kommunikationsbegriff betrifft, so geht das Arbeitspapier der Konferenz ${ }^{13}$ zwar von einer weiten Fassung aus (... development of communications and information systems ... These certainly include but are not 
limited to the so-called mass media, both electronic and non-electronic. Also requiring attention are traditional and interpersonal communications, and the whole field of point-to-point telecommunications, the postal service, and information processing, both manual - as in library and filing systems - and automatic - as in computer communications), es gelingt aber weder dem Papier noch der Konferenz zu definieren, was der Begriff in einem jeweiligen Zusammenhang umfassen soll. De facto war die Konferenz dann doch - trotz aller Lippenbekenntnisse' zu einem weiten Kommunikationsbegriff bzw. der Integration von interpersoneller und Massen-, von traditioneller und technischer Kommunikation - letztlich den „Medien”, den „Massenmedien” gewidmet. Dabei handelt es sich aber nicht nur um ein linguistisch-konzeptionelles Problem. Die weiter oben angedeutete Starrheit internationaler Bürokratie, die etwa der International Telecommunication Union, der Universal Postal Union, dem Intergovernmental Bureau of Informatics etc. bestimmte Objektbereiche zuweist und diese dann zu einem gegebenen Zeitpunkt „festschreibt”, läßt wenig Raum für die Dynamik technischer oder politischer Entwicklungen bzw. sich ändernder Sichtweisen. Die UNESCO beansprucht zwar eine Art Überkompetenz für alle Kommunikationsfragen, kann diese aber weder konzeptionell noch politisch durchsetzen. (Ein solches bürokratisches „Monster” wäre m.E. auch nicht wünschenswert, wohl aber eine echte Zusammenarbeit und Integration auf Programm-Ebene.) Mit ähnlichen begrifflichen Problemen hatte ja auch der MacBride-Bericht zu kämpfen, der ebenfalls von einem außerordentlich weiten Kommunikationsbegriff ausgeht (der erste Satz des Endberichtes lautet: "Communication maintains and animates life"14), ihn aber keineswegs durchhalten kann und will.

Aber auch die Aussagen über Modelle der Entwicklung bleiben inkonsistent. Zwar heißt es im Arbeitspapier zur Frage der Kommunikationstechnik u.a.: "Social researchers now contend that the introduction of large-scale capital-intensive technologies developed in Europe, North America and Japan into poorer, less developed societies may raise more problems than it solves... The increasing interest shown in appropriate technologies partly reflects the fact that their value lies just as much in their adaptation to a social and cultural environment as in their economic viability and technical soundness." 15 Zwar besteht weitgehende Übereinstimmung darüber, daß die sog. „klassischen” westlichen Theorien über die Rolle der Massenmedien im nationalen Entwicklungsproze $B$ aus den 50er und frühen 60er Jahren 16 überholt sind, was ja auch von den Schöpfern dieser Theorien anerkannt wird - etwa von Wilbur Schramm, Autor des „Klassikers” Mass Media and National Development (Paris 1964), der im Jahre 1979 in einem Arbeitspapier für die MacBride-Kommission darüber schrieb:

"I have just had the humbling experience of re-reading a book I wrote 17 years ago.. Two development decades have failed to produce the improvements expected of them, particularly in the poorest countries and among rural people. This failure seems to be chargeable to strategy rather than tactics. The model of change that was derived mostly from the experience of the West has proved inadequate to the task in many of the developing countries, and new models - new models rather than one new model - are now evolving ${ }^{17}$.

Schramm sieht mit vielen anderen bessere Anwendungsmöglichkeiten für Kleinmedien („Little Media, z.B. Radio, Dias, Posters, Wandzeitungen, etc) und für eine 
„lokale Strategie”. Die Mehrzahl der auf der Konferenz vertretenen Regierungsvertreter sprach jedoch in erster Linie über den notwendigen Ausbau großer, konventioneller Medien - vor allem auch auf nationaler Ebene. Und auch das Arbeitspapier stellte mit großer Eindringlichkeit und Überzeugungskraft das statistische Bild des unausgewogenen Ausbaus der Medieninfrastruktur zwischen Industrie- und Entwicklungsländern dar ${ }^{18}$.

Mit diesen Ausführungen war nicht beabsichtigt, die Ernsthaftigkeit der Bemühungen dieser Konferenz in Frage zu stellen. Die genannten Aspekte illustrieren nur den Umstand, daß mit der teilweisen Abkehr von Rhetorik und der Hinwendung zu konkreten Problemlösungsversuchen die echten Probleme erst beginnen. Die Frage der zu wählenden Kommunikationstechnik nimmt hier eine wichtige Rolle ein. Die Abkehr von den "Big Media" (Schramm) ist offensichtlich bei UNESCO-Beamten und Kommunikationswissenschaftlern stärker vertreten als bei den auf der Pariser Konferenz anwesenden Politikern und Beamten aus Entwicklungs-, Planungsund Informationsministerien.

Schramm ist zı̣zustimmen, wenn er sagt, es gäbe derzeit kein einheitliches Entwicklungsmodell mehr, sondern viele verschiedene - idealerweise angepaßt an konkrete Gegebenheiten und jeweilige Niveaus sozialer, wirtschaftlicher und nationaler Entwicklung. Das Argument für Kleinmedien ist stark, und es sollte sich vom Standpunkt des Entwicklungsprozesses weiter durchsetzen. Von der Perspektive einer Neuen Weltinformations-und-kommunikationsordnung jedoch steht ein möglichst zügiger Ausbau der Medien- und Kommunikationsinfrastrukturen im Vordergrund, und das schließt „Großmedien” mit ein; denn nur damit werden die Entwicklungsländer in die Lage versetzt, ausgewogenere Kommunikationsbeziehungen mit den Industrieländern aufzunehmen. Tendenziell stand bei der Pariser Konferenz der letzte Aspekt im Vordergrund. Aus einem hochinteressanten Arbeitspapier ${ }^{19}$, das $\mathrm{m}$.W. zum ersten Mal den Versuch unternahm, eine weltweite, zahlenmäßige Bestandsaufnahme der Medienentwicklungshilfe (Medienförderung) durchzuführen, geht hervor, daß von der Gesamtsumme aller Entwicklungshilfsprogramme in Höhe von 26 Milliarden US-Dollar im Jahre 1978 nur 226 Millionen Dollar, das ist rund 1 Prozent, auf Medien- und Kommunikationsförderung entfallen ${ }^{20}$. Die Forderung nach Erhöhung dieser Förderungsmittel stand im Mittelpunkt vieler Ausführungen, und die Vertreter einer Reihe von Industriestaaten zeigten großes Verständnis dafür, ohne jedoch bereits konkrete finanzielle Zusagen zu machen ${ }^{21}$.

Die Konferenz produzierte demnach politisch zwei wichtige Ergebnisse:

a) die Akzeptanz durch die Industriestaaten, da $ß$ in den internationalen Kommunikationsbeziehungen gravierende Unausgewogenheiten bestehen, die beseitigt werden müssen; und

b) eine grundsätzliche Bereitschaftserklärung der Industrieländer, dabei auch durch finanzielle Zuwendungen mitzuwirken.

Die Frage, die sich sofort stellt und die auch bei der Pariser Konferenz breiten Raum einnahm und äußerst kontrovers diskutiert wurde, ist die nach der Form der Abwicklung dieser in Aussicht gestellten Kommunikationsförderung. Während die Entwicklungsländer tendenziell eine möglichst starke Einbindung in multilaterale Institutionen (z.B. UNESCO) forderten - ein prononcierter Vorschlag in dieser 
Richtung war die Einrichtung eines International Institute for Information and Communication Planning innerhalb der UNESCO -, wehrten sich die Industrieländer angesichts der gegebenen Mehrheitsverhältnisse gegen eine allzu enge Verknüpfung des zu schaffenden Mechanismus mit der UNESCO. Der zustande gekommene Kompromiß sieht eine Empfehlung (die Pariser Konferenz konnte ja keine bindenden Beschlüsse fassen) an die Generalkonferenz der UNESCO vor, im Rahmen der UNESCO ein Entwicklungsprogramm für Kommunikation und als Koordinationsstelle für die Projekte ein von einem Direktor geleitetes Sekretariat zu schaffen, das von einem aus 35 Mitgliedstaaten bestehenden Verwaltungsrat geführt wird. Die Mitglieder würden von der Generalkonferenz auf der Basis geographischer Ausgewogenheit und des Rotationsprinzips gewählt.

Der Grundgedanke ist der, daß die finanziellen Mittel für Kommunikations- und Medienförderung durch diese Institution verteilt werden, wobei als Finanzierungsquellen u.a. die Errichtung eines speziellen Fonds und die Abhaltung sog. „pledging conferences" erwogen werden. Eine ähnliche Einrichtung wie die hier vorgeschlagene, wenn auch nicht mit unmittelbarer Verteil-Kompetenz, funktioniert seit zehn Jahren im Bereich der katholischen Kirche: das Catholic Media Council in Aachen. Unter Auswertung wissenschaftlicher Daten prüft es vorgeschlagene Medien-Entwicklungsprojekte, stellt Kontakte her, weckt neue Initiativen und führt auch eigene Forschungen durch ${ }^{22}$.

\section{Weitere Entwicklungen im ersten Halbjabr 1980}

Im Hinblick auf Substanz und politische Relevanz war die Pariser Konferenz über Kommunikationsentwicklung im April 1980 sicherlich das wichtigste Ereignis. Darüber hinaus hat es aber auf der Studien- und Diskussionsebene, im Überbau der Debatte sozusagen, einige Entwicklungen gegeben, die im folgenden kurz beschrieben und (in den Anmerkungen) dokumentiert werden sollen.

Hier ist zunächst die Vorlage des Endberichtes der MacBride-Kommission zu nennen. Begleitet von einem großen Maß an Aufmerksamkeit in der Fachöffentlichkeit, aber auch bisweilen in grỏßen Tages- und Wochenzeitungen, und unter den Vorzeichen der mit dem Namen UNESCO verbundenen Kontroversen hat eine 15-Personen-Kommission ${ }^{23}$ unter Vorsitz des irischen Friedensnobel- und zugleich Leninpreisträgers Sean MacBride die Aufgabe übernommen, die Gesamtheit der Kommunikationsprobleme in modernen Gesellschaften zu analysieren und Empfehlungen zu erarbeiten. Daraus wird die enorme Breite des Mandates ${ }^{24}$ deutlich und die Schwierigkeit der Aufgabe.

Der Endbericht besteht aus fünf Teilen; die ersten vier ("Communication and Society", "Communication Today", "Problems and Issues of Common Concern" und "Institutional and Professional Framework") versuchen eine analytische Bestandsaufnahme bestehender Kommunikationsstrukturen; der fünfte Teil ("Communication Tomorrow") enthält Schlußfolgerungen und Empfehlungen sowie eine Auflistung von Themen, die weiteren Studiums bedürfen. Eine Wiedergabe der Kapitelüberschriften der Empfehlungen und Schlußfolgerungen vermittelt einen Eindruck von den Themen: "Strengthening Independence and Self-reliance", "Social Consequences and New Tasks", "Professional Integrity and New Standards". "Democratization of Communication" und "Fostering International Cooperation". Der 
Bericht ist weitgehend das Ergebnis eines Verhandlungsprozesses zwischen verschiedenen, oft widersprüchlichen Auffassungen, die oftmals durch Sowohl-alsauch-Formulierungen gelöst wurden, wodurch natürlich die logische Konsistenz der Argumentation leidet. Der Bericht ist in weiten Teilen ein Konsens-Dokument, in einigen Fällen wurden abweichende Meinungen von Kommissionsmitgliedern in Fußnoten festgehalten.

Von kommunikationswissenschaftlicher Perspektive sind die Ergebnisse der Kommunikationsforschung nicht immer in genügendem Ausmaß berücksichtigt worden, eine Auffassung, die auch von zwei lateinamerikanischen Kommissionsmitgliedern (Garcia und Somavia) in einer generellen Stellungnahme am Ende des Berichts festgehalten wird: "Insufficient acknowledgement is made in general of the importance research has had in making communications an issue of contemporary debate and in underpinning a number of the commission's statements. In particular, the chapter on research could have highlighted more strongly the basic importance that serious professional research will continue to have in promoting understanding of all these issues and clarifying the underlying structural phenomena"25.

Ohne jetzt im Detail auf einzelne Passagen eingehen zu können, erscheinen mir nach Lektüre des Berichtes und seiner Empfehlungen und Schlußfolgerungen einige persönliche Bemerkungen über eine mögliche Reaktion auf den Bericht sinnvoll. Die Existenz des Berichts sollte grundsätzlich positiv bewertet werden, ebenso der Mut der Kommission, eine - wie immer imperfekte - „holistische” Betrachtungsweise gewagt zu haben. Kommunikation als sozialer Prozeß bedarf bisweilen einer solchen „ganzheitlichen” Vorgangsweise und Analyse. Der Bericht sollte nicht wie ein internationales Vertragswerk Zeile für Zeile auf Widersprüche mit den Werten unserer innerstaatlichen Kommunikationsordnungen „abgeklopft” werden - natürlich würde man da hunderte von ,anstößigen" Stellen finden. Und wäre das verwunderlich bei einer Arbeit, die eine globale Sichtweise für sich beansprucht? Ganz im Gegenteil, man sollte den Bericht auf seine vom jeweiligen Betrachter als positiv einzuschätzenden Elemente hin rezipieren und könnte daraus sicherlich wertvolle Anregung und Unterstützung für eigene kommunikationspolitische Zielsetzungen ziehen. Daß es so einen Bericht überhaupt gibt, wäre schon als Ermutigung für alle jene unter uns zu bewerten, die Fragen der Kommunikation ernst nehmen - sowohl auf wissenschaftlichen als auch auf praktischen Ebenen -, aber doch häufig in ihren jeweiligen Kontexten auf Desinteresse, Ignoranz und Gleichgültigkeit gestoßen sind. Ich plädiere also dafür, das angedeutete positive StrategiePotential des Berichts nicht durch kleinliche Detail-Polemik zu verspielen.

Schließlich sei noch darauf hingewiesen, daß auch in der Entwicklung der internationalen Kommunikationsforschung in der letzten Zeit einige Akzente gesetzt worden sind. Im Februar 1980 fand am Institute of Social Studies in Den Haag ein Workshop statt, das Fragen der Rolle der Kommunikationsforschung im Zusammenhang von Kommunikationsentwicklung behandelte. Der ausgezeichnete Bericht ${ }^{26}$, der soeben versendet wurde, enthält eine Liste von 22 konkreten Empfehlungen über eine verstärkte Rolle der Kommunikationsforschung in den Debatten und Aktionen (Aktionsforschung) um Probleme der internationalen Kommunikation, die sich in etwa auf der Linie des obigen Zitats von Garcia und Somavia bewegen. Zugleich muß jedoch mit großem Bedauern festgestellt werden, daß der UNESCO-Budgetentwurf für die Jahre 1981 bis 1983 die Entwicklung des Kommunikations- 
forschungsprogrammes wiederum außerordentlich stiefmütterlich behandelt ${ }^{27}$ und im regulären Programm nur 196.500 US-Dollar vorsieht. Durch Einwirken auf unsere nationalen UNESCO-Kommissionen könnte vielleicht bis Belgrad noch eine Lobby für eine Erhöhung dieses Budgetansatzes erreicht werden ${ }^{28}$.

Schließlich sei noch zu berichten, daß die diesjährige Konferenz der International Association for Mass Communication Research (IAMCR) in Caracas (Ende August 1980) sich Fragen der internationalen Kommunikation zum generellen Leitthema gesetzt hatte. Auch die überaus erfolgreich verlaufene Konferenz "World Communications: Decisions for the Eighties", die im Mai 1980 an der Annenberg School of Communications in Philadelphia stattfand, hat Probleme der internationalen Kommunikation zum zentralen Thema gehabt. Über 500 Forscher, Politiker, Regierungsbeamte und Medienpraktiker aus 71 Ländern konnten, so war der optimistische Eindruck, wechselseitig befruchtende Beziehungen aufbauen.

\section{Schlußbemerkung}

Dinge sind also in Bewegung gesetzt worden in der letzten Zeit. Die letztlich positive Einschätzung, die aus meinen Ausführungen hervorgeht, leitet sich aus der Auffassung ab, daß zunehmendes Bewußtsein von der Bedeutung von Kommunikation der Kommunikation insgesamt (potentiell) nur dienlich sein kann. Wichtig wäre es allerdings für unsere Länder, uns weit intensiver als bisher an den angeführten internationalen Debatten zu beteiligen und auch eine Öffentlichkeit für diese Fragen herzustellen.

\section{Anmerkungen}

1 Benno Signitzer: Internationale Kommunikationsforschung: Perspektiven für Österreich. In: Hans Heinz Fabris (Redaktion): Österreichisches Jahrbuch für Kommunikationswissenschaft I/1979. Salzburg, Neugebauer 1979, S. 242. Nach Gehard Maletzke: Intercultural and International Communication. In: Heinz-Dietrich Fischer und John C. Merrill (Hrsg.): International and Intercultural Communication. New York, Hastings House 1975. S. 410 .

2 Edward Ploman listet nicht weniger als 24 zwischenstaatliche Organisationen auf, die sich mit Fragen der internationalen Kommunikation (mit-)befassen. Vgl. Edward W. Ploman: The whys and wherefores of international organisations. In: "Intermedia", London, 8. Jg., Nr. 4, July 1980, S. 6-11, hier S. 11.

3 Ploman: a.a.O., S. 6.

${ }^{4}$ Auf die vielschichtige Debatte kann hier nicht eingegangen werden. Den Begriff von der post-industriellen (bzw. nachindustriellen) Gesellschaft prägte der amerikanische Soziologe Daniel Bell: Die nachindustrielle Gesellschaft. Frankfurt 1975; vgl. auch ders.: Cultural Contradictions of Capitalism. New York, Basic Books 1978. Marc Porat führte umfangreiche Untersuchungen der US-amerikanischen „Informationsgesellschaft” durch; vgl. Marc U. Porat: The Information Economy. Definition and Measurement. Vol. 1-11, Washington, D.C., U.S. Department of Commerce, May 1977; ders.: Communication Policy in an Information Society. In: Glen O. Robinson (Hrsg.): Communications for Tomorrow. Policy Perspectives for the 1980s. New York, Praeger 1978, S. 3-60. Auch der 
Endbericht der MacBride-Kommission ging auf diese Thematik ein: Many Voices, One World. Report by the International Commission for the Study of Communication Problems. London/New York/Paris, Kogan Page/Unipub/Unesco 1980, S. 227-229. Für eine Anwendung dieses Konzeptes auf andere Länder (hier: Österreich) vgl.: Ingo Schmoranz (Projektleiter): Makroökonomische Analyse des Informationssektors. Wien, Institut für Höhere Studien und Wissenschaftliche Forschung, Juni 1979 (2 Bände). Für eine kritische Auseinandersetzung sowohl mit dem Konzept als auch mit den Begriffen „postindustrielle Gesellschaft” und „Informationsgesellschaft” siehe Kevin Robins und Frank Webster: Information as a social relation. In: „Intermedia”, 8. Jg., Nr. 4, July 1980, S. 30-35. Eine Behandlung der Frage, inwieweit die Medien als Sektor der Volkswirtschaft angesehen werden können, unternimmt Eberhard Moths: Medien als einzel-und gesamtwirtschaftliches Phänomen. In: „Media Perspektiven”, Nr. 8, 1978; vgl. dazu auch (für Österreich) Hans Heinz Fabris und Benno Signitzer: Die österreichische Medienwirtschaft. In: „Information und Meinung", St. Pölten, 11. Jg., Nr. 2, 1980, S. 32-36.

5 Ploman: a.a.O., S. 7.

$6 \mathrm{Vgl}$. etwa aus jüngster Zeit Aufsätze wie John A. Lent: The Missionary Press of Asia, 1550-1860. In: CS, 13. Jg., Nr. 2, April-Juni 1980, S. 119-141; Ambros Eichenberger: Dritte-Welt-Filmschaffen für die Entwicklungsarbeit? In: CS, 13. Jg., Nr. 1, Januar-März 1980, S. 1-11; Michael Schmolke: Christliche Publizistik - Verantwortung für die internationale Kommunikation. In; CS, 12. Jg., Nr. 1, Januar-März 1979, S. 1-12; Max PereaExpinosa: Zur Tätigkeit der Radioschulen in Lateinamerika. In: CS, 11. Jg., Nr. 4, OktoberDezember 1978, S. 255-271; Antonio Stefanizzi: Die Informationsfreiheit heute. In: CS, 11. Jg., Nr. 3, Juli-September 1978, S. 175-186; Franz-Josef Eilers: Überlegungen zur Kommunikationsplanung in der „Dritten Welt”. In: CS, 11. Jg., Nr. 3, Juli-September 1978, S. 204-210.

$7 \mathrm{Vgl}$. etwa Jörg Becker (Hrsg.): Free Flow of Information. Informationen zur neuen internationalen Informationsordnung (= „medium"-Dokumentation 8). Frankfurt 1979; oder die „medium”-Themenhefte „Neue Weltinformations-UN-Ordnung” (9. Jg., Nr. 2, Februar 1979) und „Zerr-Perspektiven: z.B. Lateinamerika” (10. Jg., Nr. 6, Juni 1980).

8 Beispiele: Hans Bohrman u.a. (Hrsg.): Informationsfreiheit. Free Flow of Information. München, Öhlschläger 1979; „Rundfunk und Fernsehen”, 28. Jg., Nr. 1, 1980, Sonderheft zum Thema „Medien und Dritte Welt”; Jörg Becker: Die internationale Medienpolitik der Bundesrepublik zwischen Prinzipien und Interessen. In: „Rundfunk und Fernsehen”, 28. Jg., Nr. 2, 1980, S. 224-234; Werner Maier und Michael Schanne: Nachrichtenagenturen und globales Schichtungssystem. Eine Forschungsperspektive. In: „Publizistik”, 24. Jg., Nr. 2, 1979, S. 213-222; Hansjürgen Koschwitz: Internationale Publizistik und Massenkommunikation. Aufriß historischer Entwicklungen und gegenwärtiger Trends. In: „Publizistik", 24. Jg., Nr. 4, 1979, S. 458-483; Hansjoachim Höhne: Nachrichtenangebot im Wandel. In: „Publizistik”, 25. Jg., Nr. 1, 1980, S. 75-87.

9 Für eine „klassische” Ausformulierung der Forderungen der Entwicklungsländer nach einer neuen Weltinformations- und -kommunikationsordnung siehe M. Masmoudi: The new world information order. Arbeitspapier Nr. 31 der MacBride-Kommission. Paris, UNESCO, o.J. (1978-1979).

10 Näheres über die UNO-Verhandlungen über das Satellitendirektfernsehen siehe Kathry M. Queeny: Direct Broadcast Satellites and the United Nations. Alphen aan den Rijn (NL): Sijthoff \& Noordhoff 1978; vgl. auch eine Serie von Beiträgen über "Satellite Broadcasting and Communication Policy", die in Jg. 30, Nr. 2, Spring 1980 der Zeitschrift "Journal of Communication" (Philadelphia) erschienen sind; ein diesbezüglicher Beitrag über Österreich ist: Benno Signitzer: Austria: Policy-Making in a Small Country. In: "Journal of Communication", 30. Jg., Nr. 2, Spring 1980, S. 186-189. Hinsichtlich der Position Österreichs heißt es hier: "From the beginning, Austria has by and large identified herself with 
proposals developed cooperatively by Canada and Sweden that occupy a middle ground between the Western position of 'as little control as possible' and the Eastern (and partially Third World) emphasis on regulation, even of broadcasting content" (S. 188). Weitere Literatur zur spezifischen Position Österreichs: Helmut Kramer: Österreich im internationalen System. Wien, Institut für Höhere Studien und Wissenschaftliche Forschung 1978 (=1. Zwischenbericht; Studie im Auftrag des Bundesministeriums für Wissenschaft und Forschung): Hans Heinz Fabris: Kulturelle Identität und Medieneinfluß. Auslandsabhängigkeit bedroht die kulturelle Entwicklung Österreichs. In: „Die Republik”, Wien, Nr. 4, 1977, S. 12-18; Benno Signitzer: Österreich im internationalen Mediensystem. In: „Neue Entwicklungspolitik", Wien, 5. Jg., Nr. 1, 1979, S. 45-49; Institut für Publizistik und Kommunikationswissenschaft der Universität Salzburg: Österreich im internationalen Mediensystem: Beziehungen und Abhängigkeiten. Unveröff. Manuskript, Salzburg 1980.

11 Vg1. dazu Schmolke: a.a.O, hier S. 7-8. Für den vollen Text der UNESCO-Mediendeklaration siehe CS, 12. Jg., Nr. 1, Januar-März 1979, S. 60-64.

12 Many Voices, One World, a.a.O.

13 UNESCO: Intergovernmental Conference for Co-operation on Activities, Needs and Programmes for Communication Development (Paris, 14-21 April 1980), Working Paper, UNESCO-Doc. CC-80/CONF. 213/3. Paris, UNESCO, 28 February 1980, S. 2.

14 Many Voices, One World, a.a.O., S. 3. Der ganze erste Absatz, der hier zitiert wird, um die Breite des MacBride'schen Kommunikationsbegriffes, aber auch um die „Stimmung” der UNESCO-Sprache zu illustrieren, lautet: "Communication maintains and animates life. It is also the motor and expression of social activity and civilization: it leads people and peoples from instinct to inspiration, through varietated processes amd systems of enquiry, command and control; it creates a common pool of ideas, strengthens the feeling of togetherness through exchange of messages and translates thought into action, reflecting every emotion and need from the humblest tasks of human survival to supreme manifestations of creativity - or destruction. Communication integrates knowledge, organization and power and runs as a thread linking the earliest memory of man to his noblest aspirations through constant striving for a better life. As the world has advanced, the task of communication has become ever more complex and subtle - to contribute to the liberation of mankind from want, oppression and fear and to unite it in community and communion, solidarity and understanding. However, unless some basic structural changes are introduced, the potential benefits of technological and communication development will hardly be put at the disposal of the majority of mankind." (S. 3)

15 UNESCO (Working Paper), a.a.O., S. 12.

16 Für eine übersichtliche Zusammenfassung dieser „klassischen” Theorien siehe Frederick W. Frey: Communication and Development. In: Ithiel de Sola Pool u.a. (Hrsg.): Handbook of Communication. Chicago, Rand McNally 1973, S. 337-432.

17 Wilbur Schramm: Mass Media and National Development - 1979. Arbeitspapier Nr. 42 der MacBride-Kommission. Paris, UNESCO, o.J. (1978-1979), S. 1; vgl. auch den Beitrag von Rogers, einem der Begründer der sog. Diffusionstheorien: E. M. Rogers: Communication and Development. Critical Perspectives. Beverly Hills, Sage 1976. Für eine umfassende Kritik siehe Göran Hedebro: Communication and Social Change in Developing Nations. A Critical View. Stockholm, Stockholm School of Economics 1979) = Studies in Economic Psychology 110); Vgl. auch Georg-Michael Lykens: 25 Jahre "Communication and Development” - Forschung in den USA: Wissenschaft oder Ideologie? In' „Rundfunk und Fernsehen", 28. Jg., Nr. 1, 1980, S. 110-122.

18 UNESCO (Working Paper), a.a.O., S. 6-9. Vgl. auch UNESCO: Intergovernmental Conference for Co-operation on Activities, Needs and Programmes for Communication Deve- 
lopment (Paris, 14-21 April 1980), Basic Statistics on Communication Development, UNESCO-Doc. CC-80/CONF. 212/4. Paris, UNESCO, 31 March 1980.

19 UNESCO: Intergovernmental Conference for Co-operation on Activities, Needs and Programmes for Communication Development (Paris, 14-21 April, 1980), Technical Cooperation and Communication Development, UNESCO-Doc. CC-80/CONF. 212/5. Paris, UNESCO, February 1980.

20 Ebd., S. 14. Vgl. auch Klaus Winckler: Medienförderung in der Dritten Welt. In: „Rundfunk und Fernsehen", 28. Jg., Nr. 1, 1980, S. 70-91.

21 Einige kleine Industrieländer, wie z.B. die Niederlande, dagegen, kündigten bereits bestimmte Summen für diesen Zweck als Hilfe an.

22 Vgl. dazu den vorläufigen Endbericht der Pariser Konferenz: UNESCO: Intergovernmental Conference for Co-operation on Activities, Needs and Programmes for Communication Development (Paris, 14-21 April 1980), Draft Final Report, UNESCO-Doc. CC-80/ CONF. 212/8. Paris: UNESCO, 21. April 1980. - Zum Catholic Media Council vgl. Karl. R. Höller: Publizistische Medienplanung für Entwicklungsländer "Catholic Media Council", in: CS 5. Jg. 1972, S. 57-63.

23 Für Kurzbiographien der Mitglieder der MacBride-Kommission siehe: Membership of the International Commission for the Study of Communication Problems. Arbeitspapier Nr. 1 (rev. Version) der MacBride-Kommission. Paris, UNESCO, o.J. (1977/78/79).

24 Für eine Beschreibung der Aufgabenstellung der MacBride-Kommission siehe: Origin and Mandate. Arbeitspapier Nr. 2 der MacBride-Kommission. Paris, UNESCO, o.J. (1977/ 78/79).

25 Many Voices, One World, a.a.O., S. 281.

26 Communications Research in Third World Realities. The Report of a Policy Workshop held at the Institute of Social Studies, The Hague, 4-22 February 1980. Den Haag: Institute of Social Studies, o.J. (June 1980).

27 UNESCO: Draft Programme and Budget for 1981-1983, UNESCO Doc. 21C/5 (General Conference Twenty-first Session, Belgrade 1980). Paris, UNESCO 1980, S. 264.

28 Bei einem im Juni 1980 in Salzburg durchgeführten informellen Treffen der deutschen, schweizerischen und österreichischen UNESCO-Kommissionen über das UNESCOKommunikationsprogramme wurde diese Auffassung allgemein geteilt.

\section{SUMMARY}

Communications Development, the Growth of Media in Developing Countries, and the Report of the McBride Commission are frequent topics of discussion at recent international commissions. Discussion has developed, and from confrontation, progress has been made towards cooperation. This is also shown in the fact now apparently accepted that there are inbalances in the international communications relations, and also attempts to ameliorate this situation. This should be done through the development of appropriate infra-structures in Third World countries. The recommendations of the $\mathrm{McB}$ ride Report should be accepted, and a plea is made for a stronger participation of German-speaking countries in the international debate. 


\section{RÉSUMÉ}

L'article s'occupe des développements les plus récents en matière de politique internationale de communication: le développement de la communication et l'encouragement des mass media dans les pays en voie de développement, le modèle du compte-rendu final de la commission MacBride, le rôle de la recherche en matière de communication. L'auteur défend la thèse suivante: que le débat international au début des années 80 ait pu passer d'une phase de confrontation à une phase de coopération; ceci se rapporte en tout premier à la conception maintenant largement acceptée que les inégalités des rapports internationaux de communication - qui ne sont plus contestés - soient compensées par des efforts coopératifs - en particulier aussi par l'amélioration des infrastructures de communication dans les pays du tiers-monde. Plus loin, l'auteur plaide pour une réception fondamentalement positive des recommandations du compte-rendu MacBride et pour une participation renforcée des pays de langue allemande aux débats concernant la politique de communication internationale. Pour cela, la science de la publicistique et de la communication devrait jouer un rôle actif.

\section{RESUMEN}

Esta colaboraciön se ocupa de los más recientes desarrollos en el terreno de la política internacional sobre comunicación social: Desarrollo de la comunicación y promoción de los medios de difusión en los países subdesarrollados, informe final de la Comisión MacBride, papel de la investigación de la comunicación social. El autor defiende la tesis de que, con el comienzo de la década de los años 80 , el debate internacional podría pasar de una fase de confrontación a otra de cooperación. Se basa, en primera linea, en la opinión comúnmente aceptada de que las indiscutibles desigualdades en las relaciones internacionales deben ser superadas mediante cooperación internacional y, en especial, mediante la mejora de las infraestructuras en los países del Tercer Mundo. El autor aboga por la aceptación de las recomendaciones del Informe MacBride y por una mayor participación de los países de lengua alemana en los debates sobre problemas de política internacional sobre comunicación social. La publicística y las ciencias de la comunicación deben jugar un papel en este contexto. 